\title{
Erratum: Galilean invariance at quantum Hall edge [Phys. Rev. B 91, 195409 (2015)]
}

\author{
Sergej Moroz, Carlos Hoyos, and Leo Radzihovsky \\ (Received 10 March 2017; published 14 July 2017)
}

\begin{abstract}
Motivated by a recent Comment by Höller and Read [Phys. Rev. B 93, 197401 (2016)], we revisit the problem of a chiral Luttinger liquid on a boundary of a Galilean-invariant quantum Hall fluid. After correcting the linear-response calculation, the real part of the longitudinal conductivity derived in the model constructed in our paper agrees with the result found in the Comment for noninteracting fermions confined by a linear potential. We also withdraw our previous conjecture that the longitudinal conductivity contains a universal contribution determined by the "shift" and provide arguments demonstrating its nonuniversal nature.
\end{abstract}

DOI: 10.1103/PhysRevB.96.039902

In our original paper we constructed a theory of a chiral Luttinger liquid on a boundary of a Galilean invariant quantum Hall fluid. Using this theory we considered the electromagnetic response at the edge and computed the longitudinal electric conductivity in the low-frequency and small wave-vector regime,

$$
\sigma\left(\omega, p_{x}\right)=\frac{v}{2 \pi}\left(1+\frac{\mathcal{S}}{4} \frac{p_{x}^{2}}{B}\right) \frac{i c}{\omega-c p_{x}+i 0^{+}}+i m \epsilon^{\prime \prime}(B) \frac{p_{x}}{B},
$$

where we introduced the filling fraction $v$, the magnetic-field $B>0$, the velocity $c$ of the chiral edge mode, and the particle mass $m$. Note that the definition of the conductivity in this Erratum differs by an overall sign from the one used in our original paper. The present choice ensures that the real part of the conductivity is positive. The conductivity (1) differs from the conductivity extracted from the well-known edge theory of Wen [1,2] and Stone [3] by higher derivative corrections that depend on the "shift" $\mathcal{S}$ introduced in Ref. [4] and the second derivative of the energy density $\epsilon(B)$ of the quantum Hall fluid. In our paper we proposed that the first term in Eq. (1) is universal and suggested that a spectroscopic measurement of the longitudinal conductivity might be a different way to measure the shift $\mathcal{S}$ and the closely related Hall viscosity.

In a recent Comment [5] on our paper, Höller and Read computed the spectral weight (the real part of the longitudinal conductivity integrated over the frequency $\omega$ ) for the integer quantum Hall system of noninteracting fermions that occupy any number of the lowest Landau levels in the presence of an edge. For a linear confining potential, they found a result consistent with our prediction (1) except for a distinct numerical coefficient in front of the shift $\mathcal{S}$. They also demonstrated that the spectral weight depends on the functional form of the confining potential leading to the conclusion that the electromagnetic response at the edge is not universal. In the present Erratum we reexamine our fieldtheoretical analysis in light of these findings and: (i) correct our linear-response calculation and find that the numerical factor of 1/4 in the shift term of Eq. (1) should be replaced by $-1 / 2$, see Eq. (8) below. This is in agreement with Ref. [5]; (ii) confirm the nonuniversality of the coefficient of the $p_{x}^{2} / B$ term by identifying new symmetry-allowed higher derivative edge terms in the effective action.
First, we briefly summarize the improved edge field theory derived in our paper, and for details, we refer to the original paper. Our system of interest is a clean quantum Hall fluid that has translation, rotation, and Galilean space-time symmetries in the bulk. Although a generic edge breaks these symmetries, a straight edge should preserve translation and Galilean symmetries along the boundary. A bosonized field theory of a chiral Luttinger liquid living at the edge of a quantum Hall fluid was introduced by Wen [1,2] and Stone [3]. For a single chiral boson $\theta$ coupled to the electromagnetic-field $A_{\mu}$ the edge action is

$$
S_{\theta}=\frac{1}{4 \pi} \int d^{2} x\left[\frac{1}{v}\left(D_{t} \theta+c D_{x} \theta\right) D_{x} \theta-\theta E_{x}\right],
$$

where we introduced the covariant derivative $D_{\mu} \theta=\partial_{\mu} \theta-$ $\nu A_{\mu}$. The edge theory can be derived from an effective description of the quantum Hall fluid in terms of statistical gauge fields with a Chern-Simons action which we will refer to as the hydrodynamic Chern-Simons model.

Importantly, we discovered in our original paper that the edge theory is not invariant under Galilean boosts along a straight edge. In the bulk the electromagnetic response of a gapped Abelian quantum Hall fluid is encoded in the Chern-Simons theory, which is Galilean invariant. Together this implies that the total (bulk plus boundary) action is not Galilean symmetric. We thus concluded that for a Galileaninvariant quantum Hall liquid the theory (2) is incomplete and must be improved. Building on the previous work [6,7], an improved edge theory was constructed in the original paper by considering a more general problem of a quantum Hall fluid living on an arbitrary two-dimensional surface with a boundary and imposing general coordinate invariance, electromagnetic, and vielbein gauge invariance. By starting from the hydrodynamic Chern-Simons model, the resulting action for a (generically curved) edge was found to be given by

$$
S_{\theta}=\frac{1}{4 \pi} \int d^{2} x\left[\frac{1}{v}\left(\tilde{D}_{t} \theta+v^{x} \tilde{D}_{x} \theta\right) \tilde{D}_{x} \theta-\theta \tilde{\mathcal{E}}_{x}\right],
$$

with $\tilde{D}_{\mu} \theta=\partial_{\mu} \theta-\nu \tilde{\mathcal{A}}_{\mu}$. The action (3) looks almost identical to Eq. (2), but the chiral boson in the improved theory couples to the modified gauge potential $\tilde{\mathcal{A}}_{\mu}$ instead of the electromagnetic gauge potential $A_{\mu}$. In this Erratum we restrict our attention only to a quantum Hall fluid living on a flat twodimensional surface (parametrized by Cartesian coordinates) 
with a flat and static edge. In this case the modified gauge potential (up to a gauge) is given by

$$
\begin{gathered}
\tilde{\mathcal{A}}_{t}=A_{t}-\frac{m}{2} \delta_{i j} v^{i} v^{j}+\frac{s}{2} \epsilon^{i j} \partial_{i} v_{j}, \\
\tilde{\mathcal{A}}_{i}=A_{i}+m v_{i},
\end{gathered}
$$

where the parameter $s$ is proportional to the shift $\mathcal{S}$ (see below) and $v^{i}$ is the velocity of the quantum Hall fluid. In the present formulation, the velocity field is not independent but is fixed by the electric- and magnetic-fields $E_{i}$ and $B$ via the Euler equation,

$$
-m\left(\partial_{t}+v^{k} \partial_{k}\right) v_{i}=E_{i}+B v^{k} \epsilon_{k i} .
$$

In the original paper the effective theory of a chiral Luttinger liquid was organized according to the derivative expansion with the following power-counting scheme:

$$
\theta \sim \epsilon^{-2}, \quad A_{i} \sim \epsilon^{-1}, \quad A_{t} \sim \epsilon^{0} \quad \partial_{i} \sim \epsilon, \quad \partial_{t} \sim \epsilon^{2},
$$

where $\epsilon \ll 1$. This power counting is consistent with the expansion used in Ref. [6]. In this derivative expansion the Lagrangian in Eq. (2) is $O\left(\epsilon^{-1}\right)$ and will be called the leadingorder Lagrangian. On the other hand, the improved action (3) contains beyond the leading-order corrections that start with the next-to-leading $O(\epsilon)$ terms. Note also that in this power counting the edge is smooth in the following sense: The curvature of the confining potential $\partial_{y}^{2} A_{t} \sim O\left(\epsilon^{2}\right)$ is parametrically small as compared to its slope $\partial_{y} A_{t} \sim O(\epsilon)$. Here $y$ is a Cartesian coordinate parametrizing the direction perpendicular to the edge.

Using the improved edge theory (3), in our original paper we computed the longitudinal conductivity (1) at the edge. We will now compare our prediction with the results found in Ref. [5]. Consider first the case of a confining edge potential that is strictly linear. In the small wave-vector regime the real part of the longitudinal conductivity of a noninteracting integer quantum Hall fluid was found in Ref. [5] to be given by

$$
\operatorname{Re} \sigma\left(\omega, p_{x}\right)=\frac{\nu c}{2}\left(1-\frac{\mathcal{S}}{2} \frac{p_{x}^{2}}{B}\right) \delta\left(\omega-c p_{x}\right),
$$

which has the same structure as the real part of Eq. (1), but differs from it by the sign and absolute value of the numerical coefficient that multiplies the shift $\mathcal{S}$. First, we find that in order to be consistent with notations used in the original paper, the shift $\mathcal{S}=-2 s .{ }^{1}$ Since it is different from a sign from the relation we used in our paper, this fixes the sign difference between the real part of (1) and Eq. (8). Even after taking this into account, the numerical prefactors extracted from Eqs. (1) and (8) differ by a factor of $1 / 2$. We carefully revised our

\footnotetext{
${ }^{1}$ The shift $\mathcal{S}$ is defined on a sphere by $N_{\phi}=v^{-1} N-\mathcal{S}$, where $N>0$ is the particle number and $N_{\phi}$ is the magnetic flux number. Using this definition and following the arguments from Sec. 4.2 of Ref. [8], we find that the hydrodynamic Chern-Simons model Eq. (36) in our original paper fixes $\mathcal{S}=-2 s$. The parameter $s$ in our effective theory differs by a sign from: (i) the parameter $s$ introduced by Wen and Zee in Ref. [4]; (ii) the parameter $\bar{s}$ commonly used in the literature $[9,10]$. Our parameter $s$ is (plus) the "mean orbital spin per particle".
}

calculation and realized that in the original paper we have incorrectly treated the velocity field $v^{i}$ as independent when deriving the consistent current as a variation of the action with respect to the gauge field [see Eq. (46) in our paper]. In our construction, however, the velocity should not be considered independent since it is a function of the electromagnetic field satisfying Eq. (6). Taking this into account and going through the same steps as in the original paper, one recovers the result that is identical to Eq. (8).

The real part of the conductivity (8) is however not a universal result for a flat quantum Hall edge because one can write additional terms in the chiral Luttinger edge action which are consistent with symmetries (translations and Galilean boosts along the edge). As the first example, we construct a term that contributes at the next-to-leading $O(\epsilon)$ order in the derivative expansion (7) and is invariant under (time-dependent) boundary diffeomorphisms. First, we notice that any smooth spatial boundary has an associated extrinsic curvature $K_{\mu}=n_{i} \nabla_{\mu} t^{i}$ that is constructed from the normal and tangent vectors $n^{i}$ and $t^{i}$, respectively. Up to a gauge, the extrinsic curvature $K_{\mu}$ coincides with the bulk spin connection evaluated at the edge [11]. As a result, $K_{\mu}$ does not transform as a one-form under time-dependent spatial diffeomorphisms on the boundary, but can be improved to become a one-form $\tilde{K}_{t}=K_{t}-\frac{1}{2} \varepsilon^{i j} \partial_{i} v_{j}$ and $\tilde{K}_{i}=K_{i}$. Due to the presence of the vorticity, the time component of the improved extrinsic curvature is nontrivial even for a flat and static boundary. With $\tilde{K}_{\mu}$ at hand, we can construct an additional diffeomorphisminvariant contribution to the edge action,

$$
\Delta S_{\theta}=\frac{\alpha}{4 \pi} \int d^{2} x\left(\tilde{K}_{t}+v^{x} \tilde{K}_{x}\right) \tilde{D}_{x} \theta .
$$

For a flat and static boundary this term is Galilean invariant with respect to boosts along the edge and thus has to be included into the effective edge theory. Also note that since Eq. (9) is invariant on its own under electromagnetic gauge transformations, the coefficient $\alpha$ cannot be determined from effective theory considerations only but depends on the microscopic details of the edge model. Presently, the microscopic origin of this term is not understood. Importantly, the addition (9) modifies the current and the longitudinal conductivity. The resulting real part of the conductivity still has a $\delta$-function form (8) but with $\mathcal{S} \rightarrow \mathcal{S}-\alpha$.

It was argued in Ref. [5] that, if the edge mode dispersion is not strictly linear, the real part of the conductivity is not a $\delta$ function of frequency but is spread in frequency with a width of order $p_{x}^{2}$ in the limit $p_{x} \rightarrow 0$. In particular, Höller and Read [5] explicitly demonstrated that, for the integer quantum Hall problem confined by a potential that has a nonzero curvature, the edge mode dispersion is not linear and the result (8) is not valid. We note here that the correction to Eq. (8) due to the curvature of the confining potential found in Ref. [5] is of the nextto-next-to-leading order in the expansion (7) because as mentioned above in this expansion the curvature of the potential is parametrically small. This goes beyond the next-to-leading order effects we intended to incorporate in our original paper.

In a chiral Luttinger liquid edge excitations will acquire finite width as a result of interactions between chiral bosons. For example, one can start from the chiral Luttinger model (2) and introduce into the Lagrangian interaction terms between 
chiral bosons, such as $\sim\left(D_{t} \theta+c D_{x} \theta\right)^{2} D_{x} \theta$, which is of the leading order in our derivative expansion. The interaction gives rise to a self-energy that might generate nonlinear corrections to the edge dispersion and a finite decay width of chiral edge excitations. In one-dimensional Luttinger liquids this is exactly what happens if one goes beyond the Luttinger model and takes into account nonlinear corrections to the band structure close to the Fermi points which produce interaction terms, such as $\left(\partial_{x} \theta\right)^{3}[12-14]$. We emphasize that the edge model (3) derived from the general coordinate invariant hydrodynamic ChernSimons theory has no interactions between chiral bosons and thus does not lead to a finite decay width of edge excitations. We expect, however, that a generic Galilean-invariant edge theory will contain interaction terms already at the leading order in the derivative expansion, such as the one quoted above. A systematic construction of such an action will not be attempted here and is postponed to a future work.

To summarize, the Comment by Höller and Read [5] motivated us to reexamine the calculation of the longitudinal conductivity at the edge of a Galilean invariant quantum Hall fluid. After correcting our linear-response computation in our original paper, the conductivity that follows from the improved theory (3) of a chiral Luttinger liquid agrees with the result found in Ref. [5] in the case of a linear confining potential. As argued in Ref. [5], this result however is not universal and depends on the details of the edge theory. Here we presented additional arguments supporting this statement.

We acknowledge discussions with B. Bradlyn, A. Gromov, J. Höller, and N. Read. The work of S.M. was supported by the Emmy Noether Programme of the German Research Foundation (DFG) under Grant No. MO 3013/1-1. This work was partially supported by the Spanish Grant No. MINECO16-FPA2015-63667-P. C.H. was supported by the Ramon y Cajal Fellowship No. RYC-2012-10370. L.R. acknowledges support from the NSF Grant No. DMR-1001240, through the KITP under Grant No. NSF PHY-1125915 and from a Simons Investigator award from the Simons Foundation.
[1] X. G. Wen, Phys. Rev. B 43, 11025 (1991).

[2] X.-G. Wen, Int. J. Mod. Phys. B 06, 1711 (1992).

[3] M. Stone, Ann. Phys. (NY) 207, 38 (1991).

[4] X. G. Wen and A. Zee, Phys. Rev. Lett. 69, 953 (1992).

[5] J. Höller and N. Read, Phys. Rev. B 93, 197401 (2016).

[6] C. Hoyos and D. T. Son, Phys. Rev. Lett. 108, 066805 (2012).

[7] D. T. Son, arXiv:1306.0638.

[8] X.-G. Wen, Adv. Phys. 44, 405 (1995).

[9] N. Read and E. H. Rezayi, Phys. Rev. B 84, 085316 (2011).
[10] B. Bradlyn, M. Goldstein, and N. Read, Phys. Rev. B 86, 245309 (2012).

[11] A. Gromov, K. Jensen, and A. G. Abanov, Phys. Rev. Lett. 116, 126802 (2016).

[12] F. Haldane, J. Phys. C 14, 2585 (1981).

[13] K. V. Samokhin, J. Phys.: Condens. Matter 10, L533 (1998).

[14] A. Imambekov, T. L. Schmidt, and L. I. Glazman, Rev. Mod. Phys. 84, 1253 (2012). 